\title{
HOPF BIFURCATION AT INFINITY WITH DISCONTINUOUS NONLINEARITIES
}

\author{
XIANGJIAN HE ${ }^{1}$
}

(Received 3 September 1990; revised 30 November 1990)

\begin{abstract}
In this paper, we consider the existence of a family of periodic solutions of large amplitude when a pair of eigenvalues of the linear part of a first-order system of ordinary differential equations crosses the imaginary axis. We refer to this problem as a Hopf bifurcation problem at infinity. In our work, the nonlinearities may be discontinuous at the origin, and the proof of existence of periodic solutions is arrived at through the corresponding system of integral equations. The applicability of the result is demonstrated by the study of the dynamics of a train truck wheelset system.
\end{abstract}

\section{Introduction}

In recent years, many researchers have concerned themselves with the Hopf bifurcation problem of dynamical systems. The Hopf bifurcation theorem is the simplest result which guarantees the bifurcation of a family of smallamplitude time-periodic solutions of an evolution equation from a family of equilibrium solutions.

The bifurcation of periodic orbits from certain critical points of a real $n$ dimensional $(n \geq 2)$ first-order system of autonomous ordinary differential equations was treated by E. Hopf [1] in 1942. To explain briefly Hopf's work, let the differential equation be denoted by

$$
d x / d t=F(\lambda, x), \quad x \in R^{n},
$$

where $\lambda$ is a real parameter, and let $x^{\lambda}$ be a critical point at $\lambda$. Let it be assumed that $F$ is analytic in a neighbourhood of $(\lambda, x)=\left(0, x^{0}\right)$ and let

\footnotetext{
${ }^{1}$ School of Information Sc. and Tech., Flinders University of S. A., Adelaide S.A., Australia.

(C) Copyright Australian Mathematical Society 1991, Serial-fee code 0334-2700/91
} 
the matrix $F_{x}\left(0, x^{0}\right)$ have exactly two, nonzero, purely imaginary eigenvalues, say $\pm i \omega_{0}$, and no eigenvalues of the form $0, \pm 2 i \omega_{0}, \pm 3 i \omega_{0}, \ldots$ Hopf proved that a nonconstant periodic orbit bifurcates from $(\lambda, x)=\left(0, x^{0}\right)$ under the sole additional assumption that $\alpha^{\prime}(0) \neq 0$, if $\alpha(\lambda)+i \omega(\lambda)$ denotes that eigenvalue of $F_{x}\left(\lambda, x^{\lambda}\right)$ which is a continuous extension of $+i \omega_{0}$. Using a different approach, Friedrichs [2] established an existence theorem for the two-dimensional problem under the assumption that $F$ in (1.1) is only three times continuously differentiable. Friedrichs' other assumptions are in a slightly different, though equivalent, form. Several other authors have developed the theory in various directions using varying assumptions. Relaxing the condition $\alpha^{\prime}(0) \neq 0$ and requiring that the critical point $x^{0}$ of (1.1) at $\lambda=0$ be asymptotically stable, Chafee [3] has shown the existence of two manifolds of bifurcating periodic orbits which are at times distinct. Under certain simplifying assumptions on the form of $F$ in (1.1), Brushinskaya [4] has established criteria sufficient to guarantee that the bifurcated periodic orbit exists. In [5, 6], the authors proved the Hopf bifurcation theorem specially suited to systems in block-diagram form by the method of harmonic balance. Some other authors, following Hopf, have approached the bifurcation problem by trying to vary the initial conditions and parameters so as to produce a nontrivial time periodic solution (see, for example, $[7,8]$ ). Others have introduced the unknown period explicitly as a new parameter in the equations and attempted to find solutions having a known period (see, for example, [9-11]). It is difficult to compare those papers since they are set in different technical frameworks and have related but differing hypotheses. But no matter what they did, they all required that the nonlinearities be of $C^{1}$ class. In [12], employment of topological considerations made it possible to throw aside the usual assumptions of differentiability of nonlinear terms, but the continuity near the origin was still necessary for the discussion.

In this paper, we drop this assumption of continuity at the origin and examine large periodic solutions. To this end, we consider what happens if a pair of eigenvalues of the linear part of the right hand side in (2.1) below crosses the imaginary axis for large $|x|$. We refer to this problem as Hopf bifurcation at infinity, following Glover [13].

In Section 2, we construct a sequence of continuous operators, which converges to the given jump nonlinearity. For each operator in this sequence, using Glover's terminology [13] along with the method of parameter functionalisation [12], we establish when the corresponding system with the continuous nonlinearity has a periodic solution with large amplitude. We prove that the limit of a subsequence of these solutions is a nonzero, periodic solution of the system with the jump nonlinearity. 
Furthermore, if we replace the condition (2.2) by

$$
\lim _{\|x\| \rightarrow 0} \frac{\|a(\lambda, x)\|}{\|x\|}=0
$$

then the existence of small periodic solutions can be discussed in the same way. So, our existence result stated in Theorem 2.1 of this paper is clearly related to several previous works in [2-13]. One will find that our result is stronger than those in [2-13].

In order to show the applicability of the result, a train truck wheelset system of Hadden [14] considered by Glover [13] is re-examined here. For such wheelsets, many researchers [13-17] have concerned themselves with the existence of the periodic solutions. In many instances, the proofs are not rigorous. For example, in [14], the authors try to find the periodic solutions using the describing function representation and ignoring higher order harmonics, and in [13, 17], Glover tries to verify the existence of the periodic solutions of a wheelset by replacing the discontinuous nonlinearities (sgn functions) by tanh functions, which are continuous. One can easily see that the existence results in $[13,14,17]$ cannot guarantee the existence of the periodic solutions of a wheelset and our result of a wheelset dynamics in this paper implies the result in [13].

\section{Main result and its proof}

Let us consider the system

$$
d x / d t=A(\lambda) x+a(\lambda, x),
$$

where $A(\lambda)$ is a linear operator defined on $R^{n}$ and takes values in $R^{n}$, $a(\lambda, x)$ is an operator from $R \times R^{n}$ to $R^{n}$ and where $\lambda$ is a parameter. We make two assumptions:

(HA)(i) $A\left(\lambda_{0}\right)$ has exactly two, nonzero, purely-imaginary eigenvalues, say $\pm i \omega_{0}\left(\omega_{0} \neq 0\right)$

(ii) $A\left(\lambda_{0}\right)$ has no eigenvalues of the form $0, \pm 2 i \omega_{0}, \pm 3 i \omega_{0}, \ldots$;

(iii) let $p(\lambda)$ be the continuous branch of eigenvalues of $A(\lambda)$ passing through the eigenvalue $i \omega_{0}$ for $\lambda=\lambda_{0}$. Then the real part, $\operatorname{Re}(p(\lambda))$, takes values of opposite sign in every neighbourhood of $\lambda_{0}$. 
(Ha)(i) $a(\lambda, x)$ is a bounded operator,

(ii) $a(\lambda, x)$ is continuous in $\lambda$;

(iii) $a(\lambda, x)$ is piecewise continuous in $x$;

(iv) $a(\lambda, x)$ obeys the growth condition

$$
\lim _{\|x\| \rightarrow \infty} \frac{\|a(\lambda, x)\|}{\|x\|}=0
$$

uniformly with respect to $\lambda$.

We shall now state the main result of this paper:

THEOREM 2.1. If $(H A)$ and $(\mathrm{Ha})$ are true, then for every $\varepsilon>0$ there exist $a \lambda_{\varepsilon}$ in the interval $\left(\lambda_{0}-\varepsilon, \lambda_{0}+\varepsilon\right)$, a constant $C_{\varepsilon}>0$ and a corresponding nonzero continuously $T_{e}$-periodic vector function $x_{\varepsilon}(t)\left(\left|T_{\varepsilon}-2 \pi / \omega_{0}\right|<\varepsilon\right)$ such that

and

$$
12 C_{e} \geq\left\|x_{e}(t)\right\| \geq C_{\varepsilon}>\frac{1}{\varepsilon} \quad(-\infty<t<+\infty)
$$

$$
x_{\varepsilon}(t)=x_{\varepsilon}\left(T_{\varepsilon}\right)+\int_{0}^{t}\left[A\left(\lambda_{\varepsilon}\right) x_{\varepsilon}(s)+a\left(\lambda_{\varepsilon}, x_{\varepsilon}(s)\right)\right] d s
$$

Proof.

STEP 1. Since $a(\lambda, x)$ is a bounded, piecewise continuous operator, we can construct a sequence $\left\{a_{k}(\lambda, x)\right\}$ of continuous operators such that

$$
a_{k}(\lambda, x) \rightarrow a(\lambda, x)
$$

for each $x \in R^{n}$ and $\lambda \in R$ evidently. Without loss of generality, we can assume that there exists a positive number $M \in R$ such that

$$
\left\|a_{k}(\lambda, x)\right\| \leq M
$$

for all $x \in R^{n}$ and $\lambda \in R$.

STEP 2. Let $P$ denote the projection onto the two dimensional, real subspace spanned by the eigenvectors of $\pm i \omega_{0}$. Perform the change of variable from $x$ to $u$ through [13]:

$$
u=x /\|P x\|^{2}
$$

Then the system

$$
d x / d t=A(\lambda) x+a_{k}(\lambda, x)
$$

is equivalent to a new dynamical system given by [13]

$$
d u / d t=M(\lambda) u+b_{k}(\lambda, u),
$$

when $P x$ or $P u \neq 0$.

In (2.6),

$$
M(\lambda)=A(\lambda)-2 \operatorname{Re}(p(\lambda)) I
$$


and

$$
b_{k}(\lambda, u)=\|P u\|^{2} a_{k}(\lambda, x(u))-2\left\langle P u, a_{k}(\lambda, x(u))\right\rangle u,
$$

where $\langle\cdot, \cdot\rangle$ is the standard inner product and [13]

$$
x(u)=u /\|P u\|^{2} \text {. }
$$

By Step 1, we know that

$$
\left\|b_{k}(\lambda, u)\right\| \leq M\|P u\|^{2}+2 M\|P u\|\|u\| \leq 3 M\|u\|^{2} .
$$

STEP 3. Let $C\left([0, \kappa], R^{n}\right)$ denote the Banach space of continuous $R^{n}$-valued functions defined on the interval $[0, \kappa]$, where $\kappa=2 \pi / \omega_{0}+1$, with the topology of uniform convergence. Consider in $C\left([0, \kappa], R^{n}\right)$ the operators of the form

$$
U_{k}(T, \lambda ; u)(t)=e^{M(\lambda) t} u(T)+\int_{0}^{t} e^{M(\lambda)(t-s)} b_{k}(\lambda, u(s)) d s, \quad k=1,2, \ldots,
$$

which depend on the two parameters $T$ and $\lambda,[12]$.

Represent $U_{k}(T, \lambda ; u)$ in the form of a sum

$$
U_{k}(T, \lambda ; u)=V(T, \lambda) u+v_{k}(T, \lambda ; u),
$$

where

$$
\begin{aligned}
V(T, \lambda) u(t) & \triangleq e^{M(\lambda) t} u(t) \\
v_{k}(T, \lambda ; u)(t) & \triangleq \int_{0}^{t} e^{M(\lambda)(t-s)} b_{k}(\lambda, u(s)) d s
\end{aligned}
$$

LEMMA 2.1. For any given $k \in\{1,2, \ldots\}$, there exist sequences $\left\{q_{m} \mid q_{m}>\right.$ $\left.0, q_{m} \in R\right\}$ independent of $k,\left\{u_{k, m} \in C\left([0, \kappa], R^{n}\right)\right\},\left\{T_{k, m} \in[0, \kappa]\right\}$ and $\left\{\lambda_{k, m} \mid \lambda_{k, m}\right.$ in the neighbourhood of $\left.\lambda_{0}\right\}$ such that, for each fixed $k$,

$$
\begin{gathered}
u_{k, m}=U_{k}\left(T_{k, m}, \lambda_{k, m} ; u_{k, m}\right), \\
T_{k, m} \rightarrow T_{k, 0} \equiv 2 \pi / \omega_{0}, \quad \lambda_{k, m} \rightarrow \lambda_{k, 0} \equiv \lambda_{0}, \quad q_{m} \rightarrow 0 \text { as } m \rightarrow \infty
\end{gathered}
$$

and

$$
\frac{1}{2} q_{m} \leq\left\|u_{k, m}\right\| \leq \frac{3}{2} q_{m}, \quad\left\|P u_{k, m}\right\| \geq\left\|(I-P) u_{k, m}\right\| .
$$

REMARK. In the proof of Lemma 2.1, which follows next, we shall show that $T_{k, 0}, \lambda_{k, 0}$ here are independent of $k$.

Proof. One can see the detail of this proof in [12]. In fact, from the assumption (HA), we have that 1 is an eigenvalue of multiplicity 2 of the linear operator $V\left(2 \pi / \omega_{0}, \lambda_{0}\right)$ and the dimension of the eigenspace of the eigenvalue 1 is $2[12$, p. 153]. Without loss of generality, one can assume that 
$\left[12\right.$, p. 155] there exist two vectors $g$ and $h$ in $R^{n}$ which are independent of $k, T$ and $\lambda$, and a function $l(T, \lambda)$ such that

$$
V(T, \lambda)(g+i h)=l(T, \lambda)(g+i h),
$$

where $l(T, \lambda)$ is the continuous branch of eigenvalues passing through the eigenvalue 1 when $(T, \lambda)=\left(2 \pi / \omega_{0}, \lambda_{0}\right)$. Next, set $E_{0}=p\left[C\left([0, \kappa], R^{n}\right)\right]$ and $E^{0}=(I-P)\left[C\left([0, \kappa], R^{n}\right)\right]$, then

$$
C\left([0, \kappa], R^{n}\right)=E_{0} \oplus E^{0} \text {. }
$$

In this case, without loss of generality one can assume that $\|u\|=\|P u\|+$ $\|(I-P) u\|$ for each $u \in R^{n}$. Let

$$
\begin{aligned}
& B_{m}=\left\{u \in E_{0}:\left\|u-q_{m} g\right\| \leq \frac{1}{4} q_{m}\|g\|\right\}, \\
& B^{m}=\left\{u \in E^{0}:\|u\| \leq \frac{1}{4} q_{m}\|g\|\right\}
\end{aligned}
$$

where $q_{m}>0$ will be defined later, and

$$
\Omega_{m}=B_{m} \oplus B^{m} \text {. }
$$

Without loss of generality, we can choose $g$ to be a unit eigenvector $g$ (i.e. $\|g\|=1$ ). Then

$$
\frac{1}{2} q_{m} \leq\|u\| \leq \frac{3}{2} q_{m} \text { for } u \in \Omega_{m},
$$

by the definitions of $B_{m}$ and $B^{m}$.

NoTE. It is evident that in $R^{2}$ a sequence of Jordan curves $\left\{\mathscr{L}_{m}\right\}$ converging to $\left(2 \pi / \omega_{0}, \lambda_{0}\right)$ exist, and the boundary $\partial B_{m}$ of the ball $B_{m}$ in the plane $E_{0}$ is a Jordan curve. Therefore, for each $m$ there exists a homeomorphism $\gamma_{m}$ of the ball $B_{m}$ onto the closure $D_{m}$ of the set bounded by $\mathscr{L}_{m}$, defined by $\gamma_{m}(u)=\left(T_{m}(u), \lambda_{m}(u)\right)$ whenever $u \in B_{m}$, such that $\gamma_{m}\left(\partial B_{m}\right)=\mathscr{L}_{m}[10,157]$.

Let us consider for each $m$ and $k$ a family of $\tau$ parameter-dependent vector fields

$$
\Psi_{k, m}(\tau, u)=R_{m}(\tau, u)+S_{m}(\tau, u)+W_{k, m}(\tau, u)
$$

where $\tau \in[0,1], u \in \Omega_{m}$ and

$$
\begin{aligned}
R_{m}(\tau, u) & =\left\{I-V\left(\gamma_{m}(P u)\right)\right\}\left\{\tau q_{m} g+(1-\tau) P u\right\}, \\
S_{m}(\tau, u) & =\left\{I-V\left(\tau \gamma_{0}+(1-\tau) \gamma_{m}(P u)\right)\right\}(I-P) u, \\
W_{k, m}(\tau, u) & =-(1-\tau) v_{k}\left(\gamma_{m}(P u), u\right), \\
\gamma_{0} & =\left(2 \pi / \omega_{0}, \lambda_{0}\right) .
\end{aligned}
$$


We should like to define $q_{m}>0$ in such a way that $\Psi_{k, m}(\tau, u) \neq 0$ for all $\tau$ from the interval $[0,1]$ and $u$ from the boundary of the cylinder $\Omega_{m}$.

Note that it is easy to verify that (see, for example, [12] pp. 158-159) there exist $m_{0}>0, \hat{r}$ and $r_{m}$, which is dependent on $m$ but independent of $q_{m}$, such that

$$
\left\|R_{m}(\tau, u)\right\| \geq \frac{3}{4} r_{m} q_{m} \quad \text { for } u \in \partial B_{m} \oplus B^{m}
$$

and

$$
\left\|S_{m}(\tau, u)\right\| \geq \frac{1}{4} \widehat{r} q_{m} \text { for } m \geq m_{0} \quad \text { and } \quad u \in B_{m} \oplus \partial B_{m} .
$$

Furthermore, we have that

$$
\begin{aligned}
\left\|W_{k, m}(\tau, u)\right\| & \leq\left\|v_{k}\left(T_{m}(P u), \lambda_{m}(P u) ; u\right)\right\| \\
& \leq 3 M\|u\|^{2} C,
\end{aligned}
$$

by the definition of $v_{k}$ in (2.10), where $C$ is a constant which is independent of $m, k, T_{m}$ and $\lambda_{m}$.

If here $u \in \Omega_{m}$, then by (2.12) $\|u\| \leq(3 / 2) q_{m}$. Therefore,

$$
\left\|W_{k, m}(\tau, u)\right\| \leq \frac{27}{4} M C q_{m}^{2} \text { for } u \in B_{m} \oplus B^{m} \text {. }
$$

Hence, we can choose $q_{m}$ in such way that the following inequalities would be fulfilled

$$
\begin{aligned}
& \left\|R_{m}(\tau, u)\right\|>\left\|P W_{k, m}(\tau, u)\right\| \text { for } \tau \in[0,1], u \in \partial B_{m} \oplus B^{m}, \\
& \left\|S_{m}(\tau, u)\right\|>\left\|(I-P) W_{k, m}(\tau, u)\right\| \text { for } \tau \in[0,1], u \in B_{m} \oplus \partial B_{m},
\end{aligned}
$$

and also the sequence $\left\{q_{m}\right\}$ can be chosen in such a way that $q_{m} \rightarrow 0$ as $m \rightarrow \infty[10$, p. 159$]$.

Thus, we know that

$$
\Psi_{k, m}(\tau, u) \neq 0 \text { for } \tau \in[0,1], u \in \partial \Omega_{m},
$$

so that $\Psi_{k, m}(0, u) \triangleq \Phi_{k, m}(u)$ has a singular point in $\Omega_{m}$ (see, for example, [12], pp. 159-160).

Note that,

$$
\begin{aligned}
\Phi_{k, m}(u) & =R_{m}(0, u)+S_{m}(0, u)+W_{k, m}(0, u) \\
& =\left\{I-V\left(\gamma_{m}(P u)\right)\right\} u-v_{k}\left(\gamma_{m}(P u), u\right) \\
& =u-\left\{V\left(T_{m}(P u), \lambda_{m}(P u)\right) u+v_{k}\left(T_{m}(P u), \lambda_{m}(P u) ; u\right)\right\} \\
& =u-U_{k}\left(T_{m}(P u), \lambda_{m}(P u) ; u\right) .
\end{aligned}
$$

Hence, there exists $u_{k, m}$ such that

$$
u_{k, m}=U_{k}\left(T_{m}\left(P u_{k, m}\right), \lambda_{n}\left(P u_{k, m}\right) ; u_{k, m}\right) \text {. }
$$


$\left\|P u_{k, m}\right\| \geq\left\|(I-P) u_{k, m}\right\|$, obviously, by the definition of $\Omega_{m}$. Let

$$
T_{k, m}=T_{m}\left(P u_{k, m}\right), \quad \lambda_{k, m}=\lambda_{m}\left(P u_{k, m}\right) \text {. }
$$

Then, for each $k$, by the definition of $\gamma_{m},\left(T_{k, m}, \lambda_{k, m}\right) \in D_{m}$. See the Note above in this step. Hence, by Lemma Al.1 in Appendix $1,\left(T_{k, m}, \lambda_{k, m}\right)$ $\rightarrow\left(T_{k, 0}, \lambda_{k, 0}\right) \equiv\left(2 \pi / \omega_{0}, \lambda_{0}\right)$, which is independent of $k$, as $m \rightarrow \infty$ for each $k$. This completes the proof of Lemma 2.1.

REMARK. By the definition of $U_{k}$ in (2.8), (2.18) means that there exists $u_{k, m} \in C\left([0, \kappa], R^{n}\right)$ such that for $k=1,2, \ldots$,

$$
u_{k, m}(t)=e^{M\left(\lambda_{k, m}\right) t} u_{k, m}\left(T_{k, m}\right)+\int_{0}^{t} e^{M\left(\lambda_{k, m}\right)(t-s)} b_{k}\left(\lambda_{k, m}, u_{k, m}(s)\right) d s,
$$

So, by the continuity of $b_{k}, u_{k, m}(t)$ is a $T_{k, m}$-periodic solution of

$$
d u / d t=M\left(\lambda_{k, m}\right) u+b_{k}\left(\lambda_{k, m}, u\right) \text {. }
$$

Hence, we have

LemMA 2.2. For any given $k \in\{1,2, \ldots\}$, there exist sequences $\left\{q_{m}>0\right\}$, $\left\{T_{k, m} \in[0, \kappa]\right\},\left\{\lambda_{k, m}\right\}$ and a sequence of $T_{k, m}$-periodic solutions $\left\{u_{k, m}(t)\right\}$ of the system (2.19) such that for this $k$,

$$
\lambda_{k, m} \rightarrow \lambda_{k, 0} \equiv \lambda_{0}, T_{k, m} \rightarrow T_{k, 0} \equiv 2 \pi / \omega_{0}, q_{m} \rightarrow 0 \text { as } m \rightarrow \infty
$$

and

$$
0<\frac{1}{2} q_{m} \leq\left\|u_{k, m}\right\| \leq \frac{3}{2} q_{m}, \quad\left\|P u_{k, m}\right\| \geq\left\|(I-P) u_{k, m}\right\| .
$$

STEP 5. Consider the solution $u_{k, m}$ shown in Lemma 2.2. Then, $P u_{k, m} \neq 0$ evidently. Let

$$
x_{k, m}=\frac{u_{k, m}}{\left\|P u_{k, m}\right\|^{2}} \text {. }
$$

Then, by $(2.20)$,

$$
\begin{aligned}
\left\|x_{k, m}\right\|=\frac{\left\|u_{k, m}\right\|}{\left\|P u_{k, m}\right\|^{2}} & =\frac{\left\|P u_{k, m}\right\|+\left\|(I-P) u_{k, m}\right\|}{\left\|P u_{k, m}\right\|^{2}} \\
& \leq \frac{2\left\|P u_{k, m}\right\|}{\left\|P u_{k, m}\right\|^{2}} \\
& =\frac{2}{\left\|P u_{k, m}\right\|} \\
& \leq \frac{4}{\left\|P u_{k, m}\right\|+\left\|(I-P) u_{k, m}\right\|} \\
& =\frac{4}{\left\|u_{k, m}\right\|} \leq \frac{8}{q_{m}}
\end{aligned}
$$


and

$$
\left\|x_{k, m}\right\|=\frac{\left\|u_{k, m}\right\|}{\left\|P u_{k, m}\right\|^{2}} \geq \frac{1}{\left\|u_{k, m}\right\|} \geq \frac{2}{3} \frac{1}{q_{m}}>0 .
$$

Hence, by the equivalence between (2.5) and (2.6), we have

LEMMA 2.3. For any given $k \in\{1,2, \ldots\}$, there exist sequences $\left\{q_{m}>0\right\}$, $\left\{T_{k, m}\right\},\left\{\lambda_{k, m}\right\}$ and a sequence of $T_{k, m}$-periodic solutions $\left\{x_{k, m}(t)\right\}$ of the system

$$
d x / d t=A\left(\lambda_{k, m}\right) x+a_{k}\left(\lambda_{k, m}, x\right)
$$

such that for this $k$,

$$
\lambda_{k, m} \rightarrow \lambda_{k, 0} \equiv \lambda_{0}, \quad T_{k, m} \rightarrow T_{k, 0} \equiv 2 \pi / \omega_{0}, \quad q_{m} \rightarrow 0 \quad \text { as } m \rightarrow \infty,
$$

and

$$
\begin{aligned}
& 0<\frac{2}{3} \frac{1}{q_{m}} \leq\left\|x_{k, m}\right\| \leq \frac{8}{q_{m}}, \\
& \left\|P x_{k, m}\right\| \geq\left\|(I-P) x_{k, m}\right\| .
\end{aligned}
$$

Hence, by the continuity of $a_{k}$ and the equivalence of the systems of differential and integral equations, we have

LEMMA 2.4. For each $k \in\{1,2, \ldots\}$, there exist sequences $\left\{q_{m}\right\}\left(q_{m}>0\right)$, $\left\{T_{k, m}\right\},\left\{\lambda_{k, m}\right\}$ and the sequence of continuously $T_{k, m}$-periodic functions $\left\{x_{k, m}(t)\right\}$ such that for this $k$,

$$
\begin{aligned}
& x_{k, m}(t)=x_{k, m}\left(T_{k, m}\right)+\int_{0}^{t}\left[A\left(\lambda_{k, m}\right) x_{k, m}(s)+a_{k}\left(\lambda_{k, m}, x_{k, m}(s)\right)\right] d s, \\
& T_{k, m} \rightarrow T_{k, 0} \equiv 2 \pi / \omega_{0}, \quad \lambda_{k, m} \rightarrow \lambda_{k, 0} \equiv \lambda_{0}, \quad q_{m} \rightarrow 0, \quad \text { as } m \rightarrow \infty
\end{aligned}
$$
and

$$
0<\frac{2}{3 q_{m}} \leq\left\|x_{k, m}\right\| \leq \frac{8}{q_{m}} .
$$

Note that the sequences $\left\{T_{k, m}\right\},\left\{\lambda_{k, m}\right\}$ and $\left\{x_{k, m}(t)\right\}$ are bounded for each $m$. Hence, there exist convergent subsequences of them for each fixed $m$. Without loss of generality, we may assume that for each $m$,

$$
T_{k, m} \rightarrow T_{m}, \lambda_{k, m} \rightarrow \lambda_{m}, x_{k, m}(t) \rightarrow x_{m}(t) \text { as } k \rightarrow \infty .
$$

Hence, we know that $x_{m}(t)$ is continuous by Lemma 2.4 and

$$
x_{m}(t)=x_{m}\left(T_{m}\right)+\int_{0}^{t}\left[A\left(\lambda_{m}\right) x_{m}(s)+a\left(\lambda_{m}, x_{m}(s)\right)\right] d s
$$


by (2.4). Note that $D_{m}$ is closed. Thus, $\left(T_{m}, \lambda_{m}\right) \in D_{m}$. This means that $\left(T_{m}, \lambda_{m}\right) \rightarrow\left(T_{0}, \lambda_{0}\right)$ as $m \rightarrow \infty$ (see Lemma A1.1 in Appendix 1).

Furthermore,

$$
0<\frac{2}{3 q_{m}} \leq\left\|x_{m}\right\| \leq \frac{8}{q_{m}},
$$

and $q_{m} \rightarrow 0$, as $m \rightarrow \infty$. Thus, for any given $\varepsilon>0$, there exists a sufficiently large $m_{0}$ such that

$$
\begin{gathered}
\lambda_{m_{0}} \in\left(\lambda_{0}-\varepsilon, \lambda_{0}+\varepsilon\right), \quad\left|T_{m_{0}}-\frac{2 \pi}{\omega_{0}}\right|=\left|T_{m_{0}}-T_{0}\right|<\varepsilon, \\
\frac{8}{q_{m_{0}}} \geq\left\|x_{m_{0}}(t)\right\| \geq \frac{2}{3 q_{m_{0}}}>\frac{1}{\varepsilon},
\end{gathered}
$$

and

$$
x_{m_{0}}(t)=x_{m_{0}}\left(T_{m_{0}}\right)+\int_{0}^{t}\left[A\left(\lambda_{m_{0}}\right) x_{m_{0}}(s)+a\left(\lambda_{m_{0}}, x_{m_{0}}(s)\right)\right] d s .
$$

Hence, for this given $\varepsilon>0$ if we let $C_{\varepsilon}=2 /\left(3 q_{m_{0}}\right)$ and denote $\lambda_{m_{0}}, T_{m_{0}}$ and $x_{m_{0}}(t)$ by $\lambda_{\varepsilon}, T_{\varepsilon}$ and $x_{\varepsilon}(t)$ respectively then the proof of Theorem 2.1 is completed.

REMARK. If $\varepsilon$ is sufficiently small, then by (2.3) the periodic solution $x_{\varepsilon}(t)$ is very large and this is what we mean by a periodic solution of large amplitude.

\section{Existence of periodic solutions of a train truck system at infinity}

Now, let us consider the wheelset system which is taken from D'Souza and Caravavatna [14]. The equations of motion are derived by considering the three degrees of freedom in the lateral, yaw, and parallelogramming directions. The wheel profile is assumed to be conical, with $\alpha$ as the half angle of the cone. The creep forces at the rail-wheel intersection are assumed to be linear functions of creep. The equations of motion are given below.

Lateral direction:

$$
\begin{gathered}
2\left(m_{w}+m_{f}\right) \ddot{y}+c_{1} \operatorname{sgn} \dot{y}+k_{1} y+4 f_{11}\left(\frac{\dot{y}}{V}+\frac{r_{0} \alpha}{V a} \dot{y}-\psi-\theta\right) \\
+\frac{4 W \alpha}{a} y+F_{1}+F_{2}=0,
\end{gathered}
$$

where the last two terms represent the flange contact forces $F_{1}$ and $F_{2}$ which are modeled here as stiff linear springs with dead band, as shown in Figure 1. 

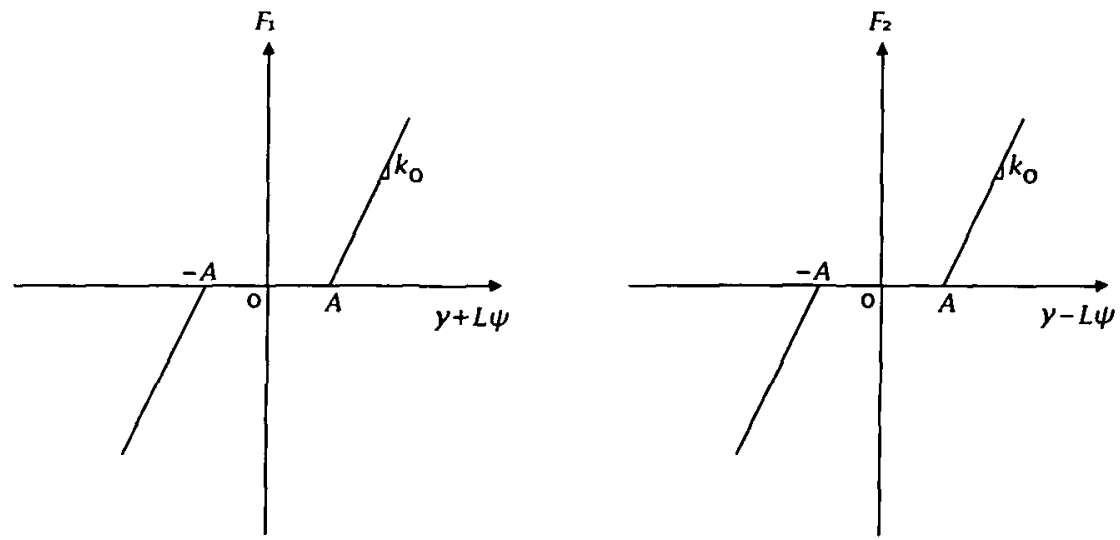

Figure 1. Flange contact forces

Yaw direction:

$$
\begin{aligned}
\left(2 I_{w}\right. & \left.+2 I_{f}+2 L^{2} m_{w}+2 d^{2} m_{f}+I_{b}\right) \ddot{\psi}+\left(2 I_{w}+I_{b}+2 d^{2} m_{f}\right) \ddot{\theta}+c_{2} \operatorname{sgn}(\dot{\psi}+\dot{\theta}) \\
& +\frac{4 f_{11}}{V} L^{2}\left(1+\frac{r_{0} \alpha}{a}\right) \dot{\psi} \\
& +4 f_{22}\left(-\frac{\alpha}{a r_{0}} y+\frac{\dot{\psi}}{V}+\frac{\dot{\theta}}{V}\right)+4 f_{33} a^{2}\left(\frac{\alpha}{a r_{0}} y+\frac{\dot{\psi}}{V}+\frac{\dot{\theta}}{V}\right) \\
& +4 W L^{2} \frac{\alpha}{a} \psi-2 W a \alpha(\psi+\theta)+F_{1} L-F_{2} L=0
\end{aligned}
$$

Parallelogramming direction:

$$
\begin{aligned}
\left(2 I_{2}\right. & \left.+2 d^{2} m_{f}+I_{b}\right)(\ddot{\psi}+\ddot{\theta})+c_{3} \operatorname{sgn} \dot{\theta}+k_{3} \theta+c_{2} \operatorname{sgn}(\dot{\psi}+\dot{\theta}) \\
& +4 f_{22}\left(-\frac{\alpha}{a r_{0}} y+\frac{\dot{\psi}}{V}+\frac{\dot{\theta}}{V}\right) \\
& +4 f_{33} a^{2}\left[\frac{\alpha}{a r_{0}} y+\left(\frac{\dot{\psi}+\dot{\theta}}{V}\right)\right]=2 W a \alpha(\psi+\theta)=0 .
\end{aligned}
$$

The parameters and typical numerical values in (3.1), (3.2) and (3.3) are listed in [14], along with the nomenclature.

Note that (see Figure 1)

$$
\begin{aligned}
F_{1} & = \begin{cases}k_{0}(y+L \psi)-A k_{0}, & y+L \psi>A \\
0, & -A \leq y+L \psi \leq A \\
k_{0}(y+L \psi)+A k_{0}, & y+L \psi<-A\end{cases} \\
& =k_{0}(y+L \psi)+ \begin{cases}-A k_{0}, & y+L \psi>A \\
-k_{0}(y+L \psi), & -A \leq y+L \psi \leq A \\
A k_{0}, & y+L \psi<-A .\end{cases}
\end{aligned}
$$


Similarly,

$$
F_{2}=k_{0}\left(y_{L} \psi\right)+ \begin{cases}-A k_{0}, & y-L \psi>A \\ -k_{0}(y-L \psi), & -A \leq y-L \psi \leq A \\ A k_{0}, & y-L \psi<-A .\end{cases}
$$

Hence, if we denote by

$$
h(z)= \begin{cases}-A k_{0}, & z>A \\ -k_{0} z, & -A \leq z \leq A \\ A k_{0}, & z<-A,\end{cases}
$$

then,

$$
\begin{aligned}
& F_{1}=k_{0}(y+L \psi)+h(y+L \psi), \\
& F_{2}=k_{0}(y-L \psi)+h(y-L \psi)
\end{aligned}
$$

and $h(z)$ is a bounded continuous vector function of $z$.

Thus, after changing variables to

$$
X=\left(x_{1}, x_{2}, x_{3}\right)^{T}=(y, \psi+\theta, \psi)^{T},
$$

(3.1), (3.2) and (3.3) become

$$
\ddot{X}+A(V) \dot{X}+(B+\delta K) X=C \operatorname{Sgn}(D \dot{X})+\delta g(X)
$$

where

$$
\begin{gathered}
A(V)=M^{-1} \frac{1}{V}\left(\begin{array}{ccc}
c_{12} & 0 & 0 \\
0 & c_{22} & 0 \\
0 & 0 & c_{33}
\end{array}\right), \quad B=M^{-1}\left(\begin{array}{ccc}
k_{11} & -k_{12} & 0 \\
-k_{21} & k_{22} & -k_{23} \\
0 & -k_{32} & k_{33}
\end{array}\right), \\
K=M^{-1}\left(\begin{array}{ccc}
2 k_{0} & 0 & 0 \\
0 & 0 & 0 \\
0 & 0 & 2 k_{0} L^{2}
\end{array}\right), \quad C=M^{-1}\left(\begin{array}{ccc}
-c_{11} & 0 & 0 \\
0 & -c_{21} & -c_{23} \\
0 & 0 & c_{23}
\end{array}\right), \\
D=\left(\begin{array}{ccc}
1 & 0 & 0 \\
0 & 1 & 0 \\
0 & 1 & -1
\end{array}\right), \\
g(X)=M^{-1}\left(\begin{array}{c}
h\left(x_{1}+L x_{3}\right)+h\left(x_{1}-L x_{3}\right) \\
0 \\
h\left(x_{1}+L x_{3}\right) L-h\left(x_{1}-L x_{3}\right) L
\end{array}\right), \\
M=\left(\begin{array}{ccc}
m_{1} & 0 & 0 \\
0 & m_{2} & 0 \\
0 & 0 & m_{3}
\end{array}\right),
\end{gathered}
$$

and the Sgn vector is given by

$$
\operatorname{Sgn}\left(x_{1}, x_{2}, x_{3}\right)=\left(\operatorname{sgn}\left(x_{1}\right), \operatorname{sgn}\left(x_{2}\right), \operatorname{sgn}\left(x_{3}\right)\right)^{\top} .
$$

In (3.4), the flange contact forces are ignored by setting $\delta=0$; otherwise $\delta=1$ and the parameters are shown in Table 1 (see Appendix 2). 
Now, we rewrite (3.4) as an equivalent first-order system by letting $Y=$ $\left(y_{1}, y_{2}, \ldots, y_{6}\right)^{\top}=\left(x_{1}, x_{2}, x_{3}, \dot{x}_{1}, \dot{x}_{2}, \dot{x}_{3}\right)^{\top}$ i.e.

$$
\dot{Y}=\left(\begin{array}{cc}
0 & I \\
-B-\delta K & -A(V)
\end{array}\right) Y+\left(C \operatorname{Sgn}\left(D\left(\begin{array}{l}
y_{4} \\
y_{5} \\
y_{6}
\end{array}\right)\right)^{0}+\delta g\left(y_{1}, y_{2}, y_{3}\right)\right) \text {. }
$$

Here, we can see that the nonlinear term of (3.6) is bounded and piecewise continuous in $y$.

A numerical calculation, using the values from Table 1 , shows that the linear matrix

$$
\left(\begin{array}{cc}
0 & I \\
-B-\delta K & -A(V)
\end{array}\right)
$$

satisfies the assumption (HA).

Let

$$
\begin{gathered}
H(V)=\left(\begin{array}{cc}
0 & I \\
-B-\delta K & -A(V)
\end{array}\right), \\
F(Y)=\left(C \operatorname{Sgn}\left(D\left(\begin{array}{l}
y_{4} \\
y_{5} \\
y_{6}
\end{array}\right)\right)^{0}+\delta g\left(y_{1}, y_{2}, y_{3}\right)\right) .
\end{gathered}
$$

Then (3.6) is equivalent to

$$
\dot{Y}=H(V) Y+F(Y) .
$$

Without loss of generality, we can assume that

(i) $H\left(V_{0}\right)$ has exactly two, nonzero, purely-imaginary eigenvalues, say $\pm i \omega_{0}\left(\omega_{0} \neq 0\right)$;

(ii) $H\left(V_{0}\right)$ has no eigenvalues of the form $0, \pm 2 i \omega_{0}, \pm 3 i \omega_{0}, \ldots$

Then, by Theorem 2.1, we have that

THEOREM 3.1. For any given $\varepsilon>0$ there exists a $V_{\varepsilon}$ in the interval ( $V_{0}-$ $\left.\varepsilon, V_{0}+\varepsilon\right)$ and a corresponding nonzero continuously $T_{\varepsilon}$-periodic vector function $Y_{\varepsilon}(t)\left(\left|T_{\varepsilon}-2 \pi / \omega_{0}\right|<\varepsilon\right)$ such that

$$
\left\|Y_{e}(t)\right\|>1 / \varepsilon \quad(-\infty<t<+\infty)
$$

and

$$
Y_{\varepsilon}(t)=Y_{\varepsilon}\left(T_{\varepsilon}\right)+\int_{0}^{t}\left[H\left(V_{\varepsilon}\right) Y_{\varepsilon}(s)+F\left(Y_{\varepsilon}(s)\right)\right] d s
$$


REMARK. If the nonlinearity $F(Y)$ in (3.7) is continuous, then for a given $V_{e}$, the vector function $Y_{\varepsilon}(s)$ shown in Theorem 3.1 is a periodic solution of the system

$$
\dot{Y}=H\left(V_{\varepsilon}\right) Y+F(Y)
$$

Hence, our result shown in Theorem 3.1 implies the result which Glover obtained in [13].

\section{Appendix 1}

LEMMA A1.1. In $R^{2}$ space, let $\left\{D_{m}\right\}$ be a sequence of bounded, closed sets. Suppose that

(i) $\mathscr{L}_{m}$ is the boundary of $D_{m}$ for each $m$ and $\left\{\mathscr{L}_{m}\right\}$ converges to $\left(T_{0}, \lambda_{0}\right)$ as $m \rightarrow \infty$, where $\left(T_{0}, \lambda_{0}\right)$ is a fixed point in $R^{2}$;

(ii) $\left\{\left(T_{k, m}, \lambda_{k, m}\right)\right\}$ is a double sequence in $R^{2}$ such that for each $m$

$$
\left(T_{k, m}, \lambda_{k, m}\right) \in D_{m}, \quad k=1,2, \ldots
$$

Then

(i) for each $k, \lim _{m \rightarrow \infty}\left(T_{k, m}, \lambda_{k, m}\right)$ exists and is equal to $\left(T_{0}, \lambda_{0}\right)$;

(ii) for each $m$, if $\lim _{k \rightarrow \infty}\left(T_{k, m}, \lambda_{k, m}\right)$ exists and is equal to $\left(T_{m}, \lambda_{m}\right)$, then $\lim _{m \rightarrow \infty}\left(T_{m}, \lambda_{m}\right)$ exists and is equal to $\left(T_{0}, \lambda_{0}\right)$.

Proof. Step 1. In fact, since $\left\{\mathscr{L}_{m}\right\}$ converges to $\left(T_{0}, \lambda_{0}\right)$ as $m \rightarrow \infty$ and $\mathscr{L}_{m}$ is the boundary of $D_{m}$, we know that each $D_{m}$ is contained in a circle with center $\left(T_{0}, \lambda_{0}\right)$ and radius $\varrho_{m}$, where $\varrho_{m} \rightarrow 0$ as $m \rightarrow \infty$. Hence, for any sequence $\left\{\left(a_{m}, b_{m}\right) \in D_{m}\right\}, \lim _{m \rightarrow \infty}\left(a_{m}, b_{m}\right)$ exists and is equal to $\left(T_{0}, \lambda_{0}\right)$. In particular, for each $k, \lim _{m \rightarrow \infty}\left(T_{k, m}, \lambda_{k, m}\right)=\left(T_{0}, \lambda_{0}\right)$.

Step 2. Since $\lim _{k \rightarrow \infty}\left(T_{k, m}, \lambda_{k, m}\right)=\left(T_{m}, \lambda_{m}\right)$ and $D_{m}$ is closed, we have that $\left(T_{m}, \lambda_{m}\right) \in D_{m}$ by (A1.1). Hence, by the statement shown in Step 1 above, $\lim _{m \rightarrow \infty}\left(T_{m}, \lambda_{m}\right)=\left(T_{0}, \lambda_{0}\right)$.

This completes the proof of this lemma. 


\section{Appendix 2}

TABLE 1. Parameter values for 80-ton hopper car truck.

$$
\begin{aligned}
& m_{1}=2\left(m_{2}+m_{f}\right)=2096 \mathrm{~kg}, \\
& m_{2}=I_{b}+2 I_{w}+2 d^{2} m_{f}=2145 \mathrm{~kg}, \\
& m_{3}=2 I_{f}+2 m_{w} L^{2}=1878 \mathrm{~kg}, \\
& c_{11}=c_{1}=23000 \mathrm{~N}, \\
& c_{12}=4 f_{11}\left(1+r_{0} \alpha / a\right)=2.103 \times 10^{7}, \\
& c_{21}=c_{2}=822 \mathrm{~J}, \\
& c_{22}=4\left(f_{22}+a^{2} f_{33}\right)=1.283 \times 10^{7}, \\
& c_{23}=c_{3}=5791 \mathrm{~J}, \\
& c_{33}=4 l^{2} f_{11}\left(1+r_{0} \alpha / a\right)=1.568 \times 10^{7} \mathrm{Nm}^{2}, \\
& k_{11}=k_{1}+4 W \alpha / a=367906 \mathrm{Nm}^{-1}, \\
& k_{12}=4 f_{11}=2.05 \times 10^{7} \mathrm{~N}, \\
& k_{21}=4\left(f_{22}-a^{2} f_{33}\right) \alpha r_{0} / a=-2.0254 \times 10^{7} \mathrm{Nm}^{2}, \\
& k_{22}=k_{3}-2 a \alpha \mathrm{W}=5.161 \times 10^{6} \mathrm{Jrad}^{-1}, \\
& k_{23}=k_{32}=k_{3}=5.17 \times 10^{6}, \\
& k_{33}=k_{3}+4 a L^{2} \mathrm{~W} / \alpha=5.180 \times 10^{6} \mathrm{Jrad}^{-1} .
\end{aligned}
$$

\section{Acknowledgements}

I would like to thank Dr. R. R. Huilgol for his interest and encouragement. Financial support was provided by The Flinders University of South Australia Research Scholarship.

\section{References}

[1] E. Hopf, "Abzweigung einer periodischen Lösung von einer stationären Lösung eines differential systems”, Ber. Sächs. Akad. Wiss. Leipzig. Math. Phys. Kl. 95 (1942) 3-22.

[2] K. O. Friedrichs, Advanced ordinary differential equations (Gordon and Breach, New York, 1965).

[3] N. Chafee, "The bifurcation of one or more closed orbits from an equilibrium point of an autonomous differential system", J. Differential Equations 4 (1968) 661-679. 
[4] N. N. Brushinskaya, "Qualitative integration of a system of $n$ differential equations in a region containing a singular point and a limit cycle", Dokl. Akad. Nauk SSSR 139 (1961) 9-12.

[5] A I. Mees and L. O. Chua, "The Hopf bifurcation theorem and its applications to nonlinear oscillations in circuits and systems", IEEE Transactions on Circuits and Systems, CAS-26 (1979) 235-254.

[6] D. J. Allwright, "Harmonic balance and the Hopf bifurcation", Math. Proc. Camb. Phil. Soc. 82 (1977) 453-467.

[7] D. Henry, "Geometric theory of semilinear parabolic equations", Lecture Notes in Math. No. 840 (1981).

[8] G. Iooss, "Existence et stabilité de la solution périodique secondaire intervenant dans les problèmes d'evolution du type Navier-Stokes", Arch. Rational Mech. Anal. 47 (1972) 301-329.

[9] P. C. Fife, "Branching phenomena in fluid dynamics and chemical reaction-diffusion theory", Proc. Symp. Eigenvalues of Nonlinear Problems (1974) 23-83.

[10] D. D. Joseph and D. H. Sattinger, "Bifurcating time periodic solutions and their stability", Arch Rational Mech. Anal. 45 (1972) 79-109.

[11] D. H. Sattinger, "Bifurcation of periodic solutions of the Navier-Stokes equations", Arch. Rational Mech. Anal. 41 (1971) 66-80.

[12] V. S. Kozjakin and M. A. Krasnosel'skii, "The method of parameter functionalization in the Hopf bifurcation problem", Nonlinear Analysis, Theory, Methods and Applications 11 (1987) 149-161.

[13] J. N. Glover, "Hopf bifurcations at infinity", Nonlinear Analysis, Theory, Methods and Applications, 11 (1989) 1393-1398.

[14] A. F. D'Souza and P. Caravavatna, "Analysis of nonlinear hunting vibrations of rail vehicle trucks", ASME, Journal of Mechanical Design 102 (1980) 77-85.

[15] F. B. Blader and E. F. Kurtz, "Dynamic stability of cars in long freight trains", $A S M E$, Journal of Engineering for Industry 96 (1974) 1159-1167.

[16] R. Hull and N. K. Cooperrider, "Influence of nonlinear wheel/rail contact geometry on stability of rail vehicles", ASME, Journal of Engineering for Industry 99 (1977) 172-185.

[17] J. N. Glover, "Bifurcations and chaos in Hadden's model of a train wheelset", M.Sc. Thesis, Dept. of Mathematics, University of Western Australia, 1989. 